\section{Multimodal therapies in canine primary immune mediated haemolytic anaemia (pIMHA): a descriptive study}

\section{Julia Tang ${ }^{1}$, Barbara Glanemann ${ }^{1}$, James Swann ${ }^{2}$}

1 The Royal Veterinary College, Hertfordshire, United Kingdom

2 Kennedy Institute of Rheumatology, University of Oxford,

Oxford, United Kingdom

\section{OBJECTIVES}

This study's aims were to describe and compare the signalment and presentation of dogs with pIMHA treated with plasmapheresis, human intravenous immunoglobulins (hIVIG), or three concurrent immunosuppressants, including outcomes of dogs in the latter category.

\section{METHODS}

A referral institution's electronic records were retrospectively searched for dogs diagnosed with pIMHA. Inclusion was based on published IMHA diagnosis guidelines. Patients which had been either treated with three concurrent immunosuppressants, received plasmapheresis or hIVIG underwent further data analysis. Descriptive statistics were used to compare among the treatment groups, and within the three concurrent drugs group.

\section{RESULTS}

Over a period of 14 years, 552 dogs were diagnosed with pIMHA. Twenty dogs received three concurrent immunosuppressive drugs, seven underwent plasmapheresis, and thirty received hIVIG. There were no significant differences regarding demographics and clinicopathological findings between groups. All dogs treated with three concurrent immunosuppressants received prednisolone as their primary therapy. The most common additional immunosuppressants were ciclosporin and azathioprine. The mean time-point for starting a second immunosuppressant was 3.15 days after commencing prednisolone (3rd immunosuppressant: 14.8 days). Eleven patients started and continued on three immunosuppressants concurrently, while nine patients began on two immunosuppressants. Seventeen patients survived up to 1 month post discharge, ten survived up to 3 months, seven up to 6 months, three were alive over 30 months and one over 90 months.

\section{STATEMENT (CONCLUSIONS)}

This study provides new information on the follow-up of pIMHA patients receiving concurrent immunosuppressants, as well as data on cases receiving plasmapheresis and hIVIG for further comparisons with conventional IMHA study populations.

\section{Diagnostic quality of bone marrow sampling and associated complications in dogs and cats}

\section{Glynn Woods, Matthew Simpson, Jasmin Paris, Craig Breheny}

Royal Dick School of Veterinary Studies, Edinburgh, United Kingdom

\section{OBJECTIVES}

To evaluate the diagnostic success of bone marrow aspiration and biopsy, the associated complications and their predisposing factors in dogs and cats.

\section{METHODS}

Single centre retrospective case review, in which clinical records were screened to identify dogs and cats that had undergone bone marrow sampling between 2012 and 2019.
Data including signalment, the presence of specific clinicopathological findings (thrombocytopenia, neutropenia, anaemia), anatomical site of bone marrow sampling, number of attempts, the diagnostic quality of sampling, analgesia protocol, and complications post-procedure were recorded.

\section{RESULTS}

There were 140 dogs and 31 cats identified that underwent bone marrow sampling. On review, 85\% (145/170) of bone marrow aspirates were of diagnostic quality and $73 \%(121 / 164)$ of bone marrow biopsies were of diagnostic quality. Bone marrow aspirate results agreed with biopsy $100 \%$ (107 cases) of the time.

Indications for bone marrow sampling included investigation of non-regenerative anaemia (29\%), oncological staging (22\%) and pancytopenia (13\%). Immune-mediated conditions accounted for $77 \%$ of all bone marrow diagnoses made (haemolytic anaemia, thrombocytopenia and neutropenia) and 23\% were neoplastic (lymphoma, leukaemia, histiocytic sarcoma).

Complications were recorded in 12\% (20/171) of cases. Pain was the most common complication of bone marrow sampling in 19/20 cases (95\%) with severe bruising reported in the remaining dog. The incidence of pain post-procedure was positively associated with the number of bone marrow sampling attempts in cats but not in dogs. 


\section{STATEMENT (CONCLUSIONS)}

Bone marrow aspirates and core biopsies agree in the majority of cases whilst complications are rare.
Post-procedural analgesia is necessary and procedurerelated haemorrhage is rare in dogs selected for bone marrow sampling, even when thrombocytopenia is present. 